\title{
DETECTING COLLISIONS AND FINDING CONTACT DURATIONS IN EXPERIMENTAL GRANULAR FLOW
}

\author{
Subodh DHAKAL ${ }^{1}$, Kazuyoshi IWASHITA ${ }^{2}$ and Masanobu ODA ${ }^{3}$ \\ ${ }^{1} \mathrm{PhD}$ student, Dept. of Civil and Environmental Engineering, Saitama University \\ (255 Shimo Okubo, Sakura ku, Saitama, Japan) \\ E-mail: s05d2057@mail.saitama-u.ac.jp \\ ${ }^{2}$ Member of JSCE, Assoc. Professor, Dept. of Civil and Environmental Engineering, Saitama University \\ (255 Shimo Okubo, Sakura ku, Saitama, Japan) \\ E-mail: iwa@mail.saitama-u.ac.jp \\ ${ }^{3}$ Member of JSCE, Professor, Dept. of Civil and Environmental Engineering, Saitama University \\ (255 Shimo Okubo, Sakura ku, Saitama, Japan) \\ E-mail: m-oda@mail.saitama-u.ac.jp
}

\begin{abstract}
With the objective of finding the promising contact detection methodology in the physical experiments, assembly of idealized disc particles with different solid fractions were sheared experimentally in the 2D shear flow apparatus. A high speed video camera and subsequent image processing techniques document the positions of the center of particles involved in the flow. This setup also provides opportunity for visual inspection of the collision time and partners. The important characteristics of pre and post collision phenomena were keenly investigated in sparse to dense particle concentration cases so that the precise criteria for each of them could be determined for distinguishing contacts. Based on these observations, new contact detection algorithm is formulated which have three components: finding potential partners of collision, detecting collision partners and finding the duration of each collision. The results obtained from proposed method are compared with the visual observations in the digital video of the physical experiment with the help of motion analytical and particle tracking PTV software. Comparisons of about hundred randomly selected data in different solid fractions and shear rate reveal that the proposed method could detect the contacts with considerable accuracy than the previous methods.
\end{abstract}

Key Words : experimental granular flow, velocity change, collision detection, contact durations

\section{INTRODUCTION}

Granular materials are the concern of many inter disciplinary field of research including civil engineering, fluid mechanics, geophysics, agriculture and chemical engineering. They are also important for geological processes where landslides, soil erosion and sedimentations determine much of the morphology of the Earth. Since the granular materials are assembly of a large number of discrete solid particles and are highly discontinuous and inhomogeneous, the deformation of the bulk of the granular materials is accomplished due to the mobilization of its constituent particles relative to each other. The inter-particle contacts are the conduits for force transmission throughout the granular mass. Therefore, the methods that deal with the bulk of the solid materials like continuum theories for granular materials ${ }^{1-3)}$ falls sort in truly characterizing the granular assembly. Precise information of the contact properties is necessary to understand the granular flow. In the physical experiments; accurate detection of contacts which is the foundation of the mechanics of granular materials, is still the major challenge. Its scope is unquestionable also in the conditions where binary and multi-particle interactions are to be separated.

In most of the previous collision-detection algorithm formulations, only the distance between particles is used to find the potential contacts ${ }^{4-6)}$. Hockney and Eastwood ${ }^{5)}$ partitioned the computational space into uniform cells where they 
considered that a particle will make contact only with the particles in the same cell or its neighboring cell in next time step. Allen and Tildesley ${ }^{6)}$ recommend using the Verlet neighbor list algorithm where they used a threshold distance to determine the radius of influence of a particle-particle interaction or the distance to search for neighbors. The neighbor lists are then needed to be updated once every several time steps. Detail review of several other methods which use the spatial subdivisions of the assembly to find the contacts especially useful in the numerical simulations can be found in Vemuri et al. ${ }^{4}$. However, it is imperative that many of the numerical simulations results in granular materials are to be validated by getting the particle scale information from the physical experiments.

Due to the development in image processing techniques, the particle positions and velocities can be obtained by direct imaging the experimental run and tracking their movement ${ }^{7-9)}$, however the data generally have limitations in terms of precision. In such conditions, the distance criteria alone might not accurately detect the contacts. In order to make more promising contact detection methods, careful investigation of the collision behavior in the experiment is more important. With this in view, the granular assembly of idealized discs is sheared in the newly designed 2D shear flow apparatus and the experimental runs are captured with the help of high speed video camera. The digital video data is later analyzed by motion analytical and particle tracking software to get the spatial position data and the trajectories. In this application, it is possible even to detect the time and partner of collision so as to observe the changes in particle movement after collision and also to validate the contact detection algorithm. In this study, it is observed that in general when particles make collision, their velocity direction changes significantly. This criterion was adopted also by Blair and Kundrolli ${ }^{7)}$ in addition to the distance between particles. However, while tracking particle movement, it is observed that if particles collide, sometimes the velocity magnitude changes significantly without substantial change in the velocity direction. Therefore, if only the velocity direction change criterion is used, many of the contacts can be easily missed. Moreover, the Blair and Kundrolli method is formulated only to find the contacts but it does not have any method to look what if the particles remain continuously in contact for long time. This is highly probable in more dense granular flow. Looking at these limitations of the previous methods of contact detection and its importance in the contact dynamics studies, the major objectives of this study is to formulate more accurate contact detection method so as it can be used to study the contact dynamics in more precise way. In this study, the criteria to detect the contacts are developed based on the observations of particle scale movement in the physical experiments.

\section{EXPERIMENTAL CONFIGURATION}

\subsection{Shear Flow Apparatus}

The shear flow apparatus consists of an inner movable wall and an outer stationary wall that rest on a bottom horizontal plate (Fig. 1). The inner and outer walls both are roughened by glued-on circular flanges of about the same radius as the particles to enhance the shear flow. The outer wall is placed on a horizontal steel plate atop several ball bearings to minimize friction between them. The inner wall velocity can be freely controlled by changing the speed of a DC motor joined to it. The spacing between the outer and inner walls, called the shear cell height, is $11.5 \mathrm{~cm}$. The distance between the upper and lower plates is $8 \mathrm{~mm}$, which is slightly larger than the particle thickness $(6 \mathrm{~mm})$. Apparatus details and physical properties of particles used in the experiments are given in Tables 1 and 2, respectively. The apparatus is covered on top with an acrylic plate to keep the particles from escaping, to generate stable flow, and to capture digital images during the experiment.

Table 1 Details of experimental shear cell apparatus.

\begin{tabular}{ll}
\hline Motor speed & $3 \sim 180 \mathrm{rpm}$ \\
Shear range, $\dot{\gamma}$ & $0.5 \sim 120 \mathrm{sec}^{-1}$ \\
Maximum torque of motor & $15 \mathrm{~N}-\mathrm{m}$ \\
Radius of inner wall & $28.5 \mathrm{~cm}$ \\
Radius of outer wall & $40 \mathrm{~cm}$ \\
Diameter of flange & $2.47 \mathrm{~cm}$ \\
No. of flanges attached to inner wall & 37 \\
No. of flanges attached to outer wall & 52 \\
\hline
\end{tabular}

Table 2. Physical properties of plastic discs.

\begin{tabular}{ll}
\hline Diameter, $D(\mathrm{~mm})$ & 23 \\
Thickness $(\mathrm{mm})$ & 6 \\
Mass $(\mathrm{g})$ & 1.127 \\
Density $\left(\mathrm{g} / \mathrm{cm}^{3}\right)$ & 0.934 \\
Coefficient of restitution, $e$ & 0.870 \\
Coefficient of friction, $\mu$ & 0.265 \\
\hline
\end{tabular}

Uniformly sized plastic discs with diameter of 2.3 $\mathrm{cm}$ and marked at the center were used in the 


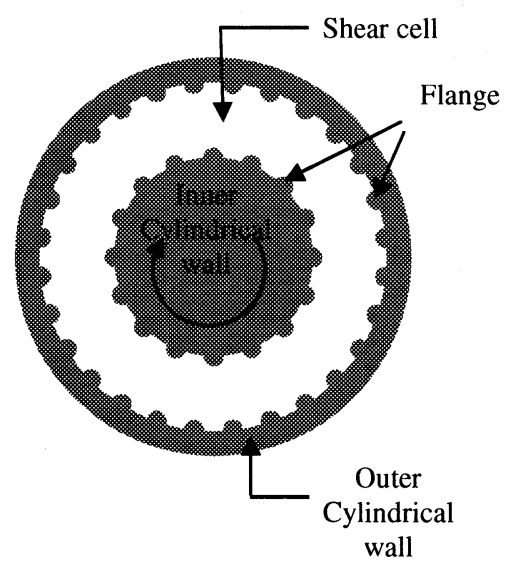

(a)

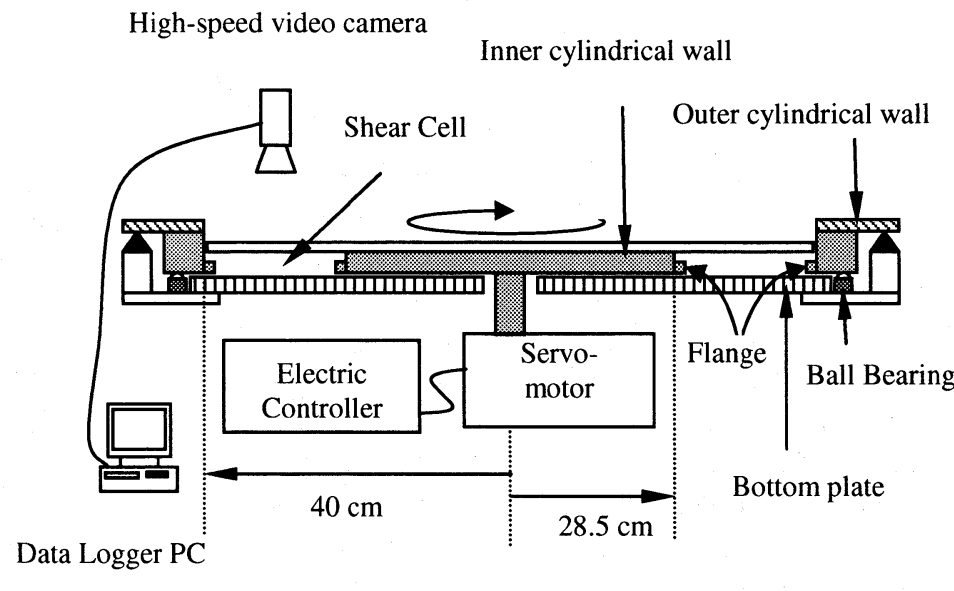

(b)

Figure 1 Schematic diagram of shear flow apparatus: a) Plan view, and b) Cross section.

experiment to permit evaluating spatial coordinates of the particle. The coefficient of friction between the particles and the shear cell floor is about 0.30 . The shear rate, $\dot{\gamma}=V_{w} / H$, (defined as the inner wall velocity $V_{w}$ divided by the shear cell height $H$ ), is changed by varying the velocity of the inner wall. The shear flow is given ample time to come to a stable state, which takes about 2 minutes after the initiation of the shearing. The stable state is judged when every particle has become part of the shear flow, and the flow is homogeneous without stoppage at any moment. After establishing the proper arrangement of the light system, the shear flow in the test section of the shear cell is recorded with the high speed video camera (FASTCAM-X 1280 PCI), which is connected to a frame grabber. The detailed specifications of the high speed video camera are given in Table 3. Each experimental run consisted of 2048 digital video frames at 1000 frames per second and a resolution of $1280 \times 512$ pixels. The recorded digital video data is later transferred to the particle tracking software to determine particles' spatial positions. These position data are used as a base data for the further data retrieving and analysis.

Table 3. Capacity of high speed video camera.

\begin{tabular}{ll}
\hline Frame rate & $60 \sim 1,000 \mathrm{~Hz}$ \\
Shutter speed & $30 \sim 10,000 \mathrm{~Hz}$ \\
Pixel resolution & $1,280 \times 512$ \\
Number of frames possible & 1000 \\
Size of the test section & $20 \times 11.5 \mathrm{~cm}^{2}$ \\
Number of pixels in one particle & 41.4 \\
Type of color & Monochrome \\
\hline
\end{tabular}

\subsection{Particle Tracking Velocimetry}

The digital video data of each experimental run captured by the FASTCAM-X 1280 PCI is transferred to the Dipp-Motion 2D motion analysis and particle tracking software produced by DITECT Co. Ltd., Japan. A sample picture of particles in the test section of the shear cell is shown in Figure 2. The particle tracking software determines the coordinates of the particles' centers, marked in black. Particle tracking software recognizes a black circular geometric shape in the image and searches for the closest matching shape to track in the following time steps. To achieve the maximum possible contrast between the floor of the shear cell and the particles, white colored plastic discs are used. The black circular marks in the center of the particles create the highest possible contrast, and the software can follow the circular mark even if the particle undergoes rotation. After tracking the positions of this particle for all the time frames, the next particle is tracked in a similar fashion.

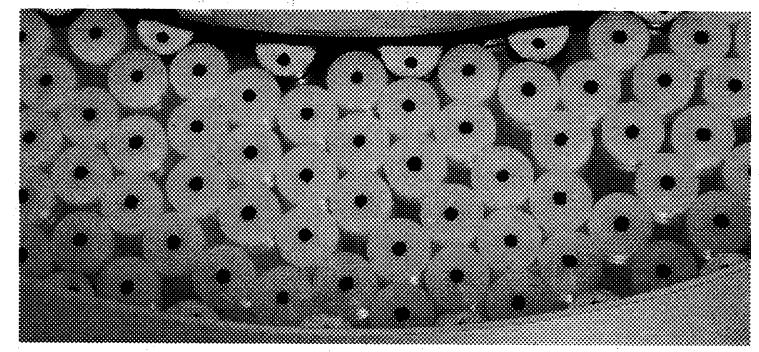

Figure 2 Sample picture of the particles in shear cell. 
After making necessary adjustments in the software and applying a conversion factor to calculate physical coordinates from pixels as given in Table 3, the spatial coordinates of the center of each particle at each frame are obtained. Other parameters, including the velocity vectors and contact properties, were determined using these spatial coordinates. To judge the accuracy of tracked data, experiments were conducted using particles that are marked at two places equidistant from the particle center and whose spatial positions are tracked. Ideally, if the data is hundred percent accurate, the distance between two mark points should be same in each of the frame owing the fluctuation distance to be zero. Experiments on about 800 randomly selected data reveals that the distance between the marks in different time steps deviates from the actual distance by $1.6 \%$ in an average (Figure 3) which is equivalent to $0.8 \%$ of the particle diameter. The standard deviation of these measurements is $0.7 \%$ of the particle diameter. Reduction of error below this limit was not possible and we need to compromise this looking at the advantage of getting particle scale information from the physical experiments of this kind. We are aware about the possibility of increasing the accuracy of the position data in the future studies.

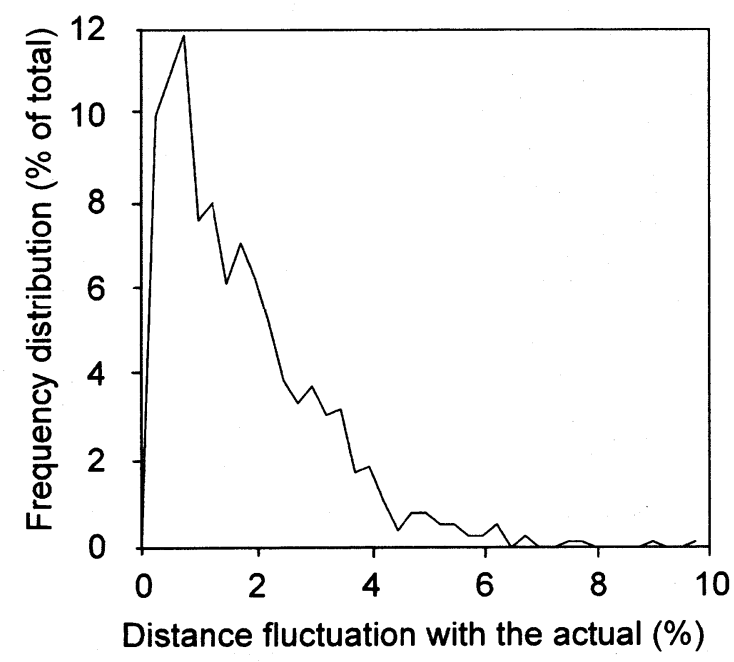

Figure 3 Frequency distribution of fluctuation of the distance between two marks in the same particle at different time frames as obtained from the particle tracking method.

\section{EXPERIMENTAL OBSERVATIONS}

To find the important pre and post collision phenomena of the inter-particle collisions, several test experiments were run and keenly investigated in the motion analytical and particle tracking software.
It is observed that after the particles undergo collision, mostly the particles suffer significant velocity direction change compared to previous time step. The twist and turns seen in trajectories of particles with shear rate of $34.6 / \mathrm{s}$ and solid fraction of 0.40 plotted in Figure 4 are found to be the result of collision.

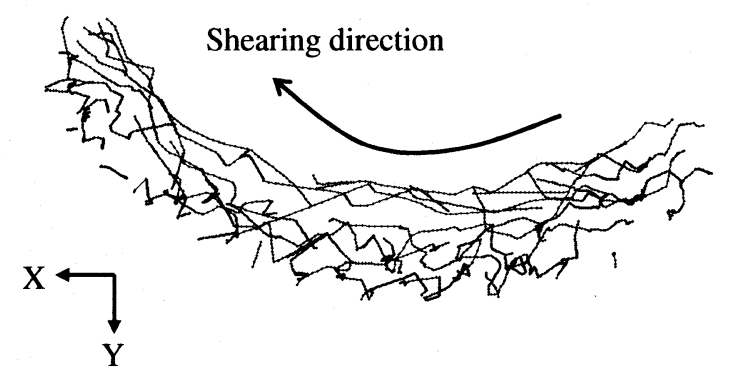

Figure 4 Typical particle movement trajectories (solid fraction of 0.40 and shear rate of $34.6 / \mathrm{s}$ ).

One of the important observations is that when one of the almost stationary particles is hit by the other moving particle from the back, it is possible that there is no change in velocity direction even after collision (Figure 5). This is particularly possible when the solid fraction is very low so that particles have enough space to travel without any disturbance. However, in this case, the momentum of one particle is transferred to the other during collision; therefore one of the particles got increase in the velocity magnitude while the other almost stops (Figure 6). In Figure 5, after Particle 2 hits the stationary Particle 1 from back, Particle 2 stops.

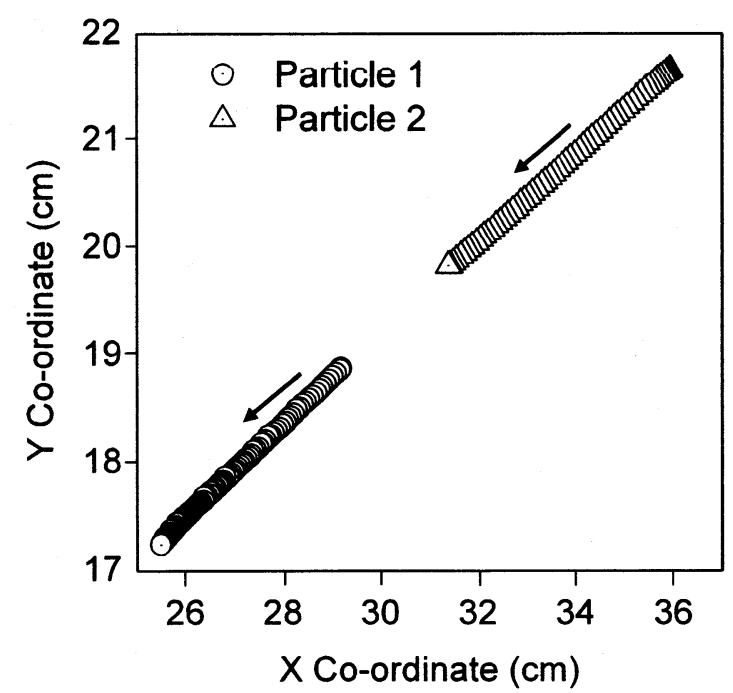

Figure 5 Locus of two particles where moving Particle2 hits the stationary Particle1 from back (very sparse case), arrow indicates the particle movement direction. 


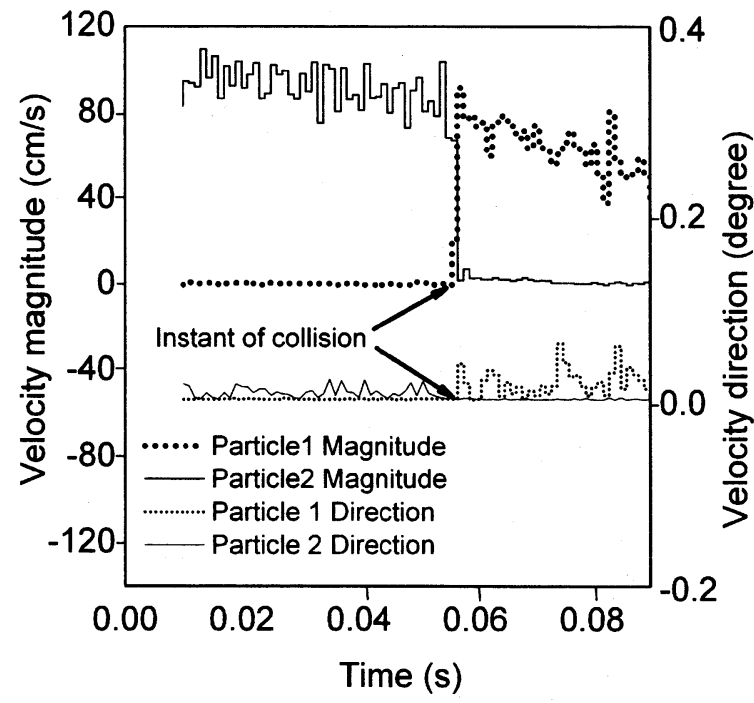

Figure 6 Temporal variation of velocity magnitude and direction of two particles where moving Particle2 hits the stationary Particlel from back (very sparse case). There is negligible direction change even after collision for this particular pair.which is supported also from Figure 5.

If both of the particles move in the same direction and one of them hit the other from back, both of the particles could experience change in velocity magnitude: increase in one and decrease in the other. If however, both of the particles move towards each other, after the collision both of them experience significant change in the velocity direction (Figure 7) in addition to the change in velocity magnitude. It is important observation to find the potential partners of collision. The distance between the particle centers can then be used to distinguish the collision partners. The next question is what will happen if both of the particles collided but they remain in contact for some more time. This can be solved by considering the relative velocity of this pair along the contact normal direction. If the particles remain in contact, they will move together and therefore the relative velocity will be zero. If the particles are not much rigid and there is possibility of overlapping, then the relative velocity along the contact normal direction will be negative. The overlapped particles will separate once the relative velocity becomes more than zero. The duration of contact can thus be calculated by subtracting the time of first collision from the time of separation. In this way criteria for detecting the contacts, and finding the contact durations can be formulated. One of the parameters that is not considered in this study is the individual particle rotations which might play role on making and destroying the contacts. However, this parameter can be adjusted in the future refinement of the collision detection and contact duration finding methodology.

\section{FORMULATION OF ALGORITHMS}

\subsection{Collision-detection algorithm}

The collision-detection algorithm adopted in this study has three major components based on the observations in the physical experiments: finding potential candidates for collision, detecting collision partners, and checking if contact is lost or continues. The flow chart for detecting collisions is shown in Figure 8(a). The flow chart for determining whether a collision vanishes or continues in the next time steps is shown in Figure 8(b), which is also part of the contact duration-finding algorithm. The criteria concerning changes in velocity vectors sorts out potential candidates for collision, whereas the criteria concerning distance between particle centers confirms the collision partners, as can be seen in Figure 8(a). Once collision is detected, the relative velocity of collision partners in the next time step is considered to check whether contact is lost or continues, as shown in Figure 8(b).

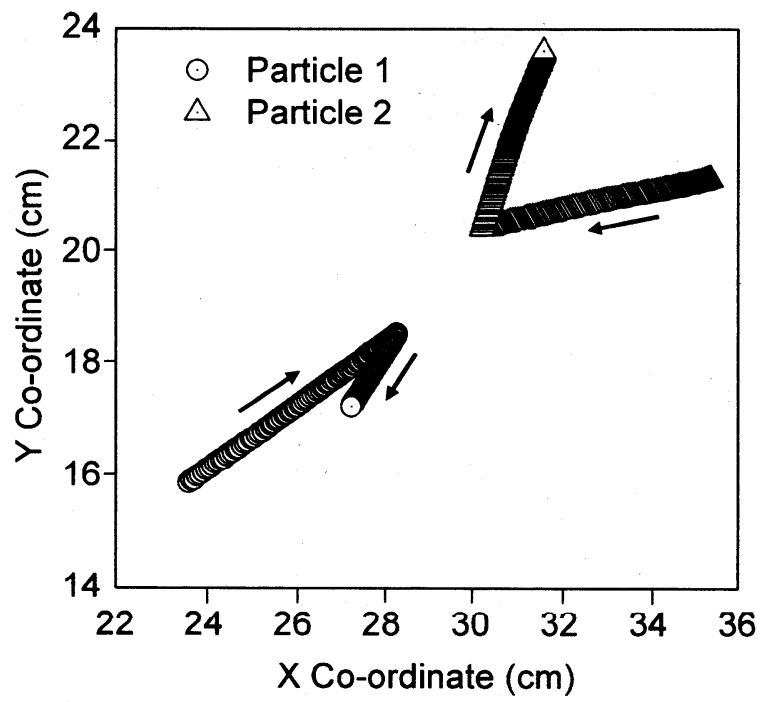

Figure 7 Locus of two particles where both the particles are moving towards each other (head on collision), arrow shows the movement direction.

\subsubsection{Finding potential candidates of collision}

To find the potential candidates of collision, the velocity vectors are determined by using the center coordinates of the particles that are obtained from the DippMotion software. The velocity of every particle in the streaming and the transverse direction is first calculated using the instantaneous position difference of the center co-ordinates of the particles 


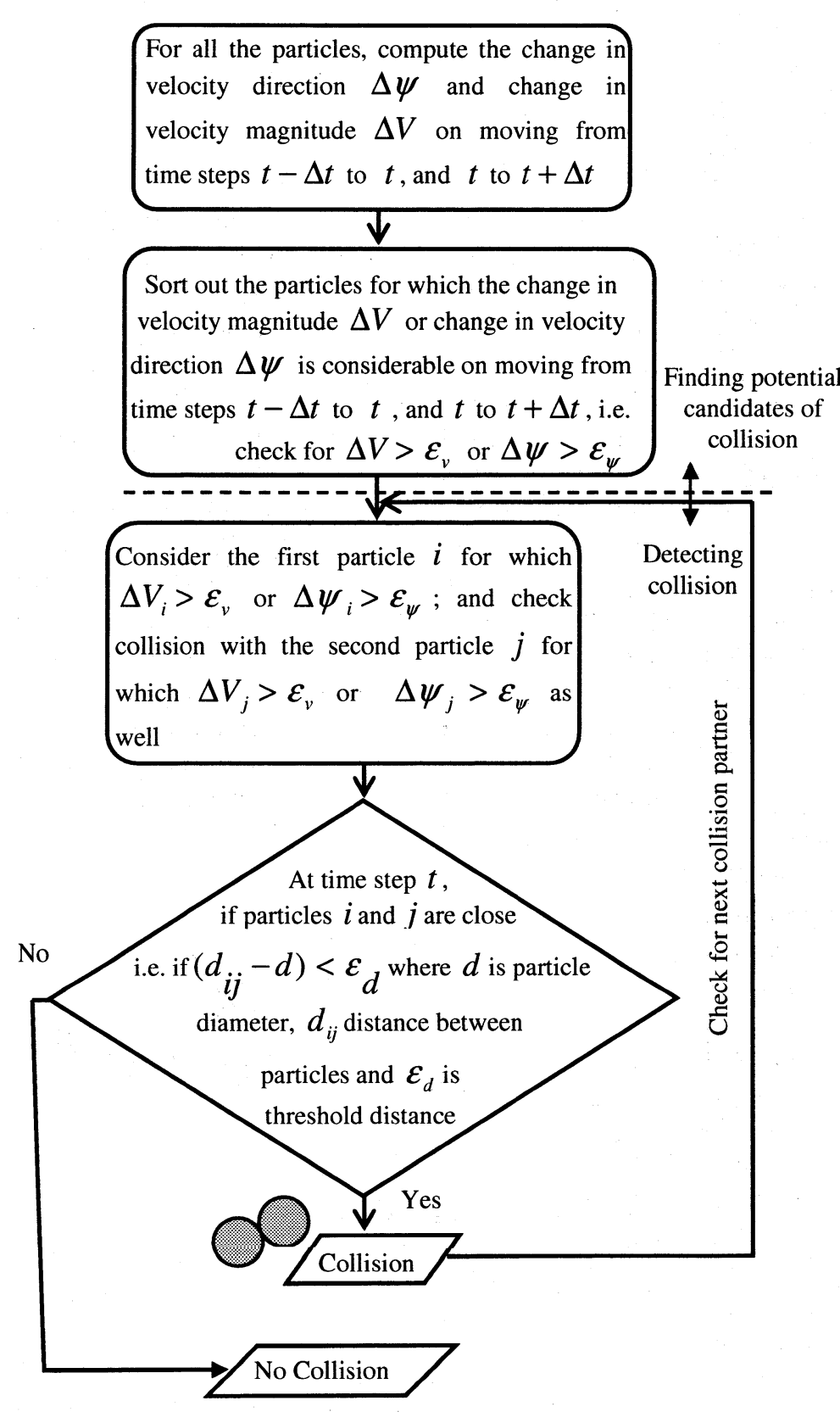

(a)

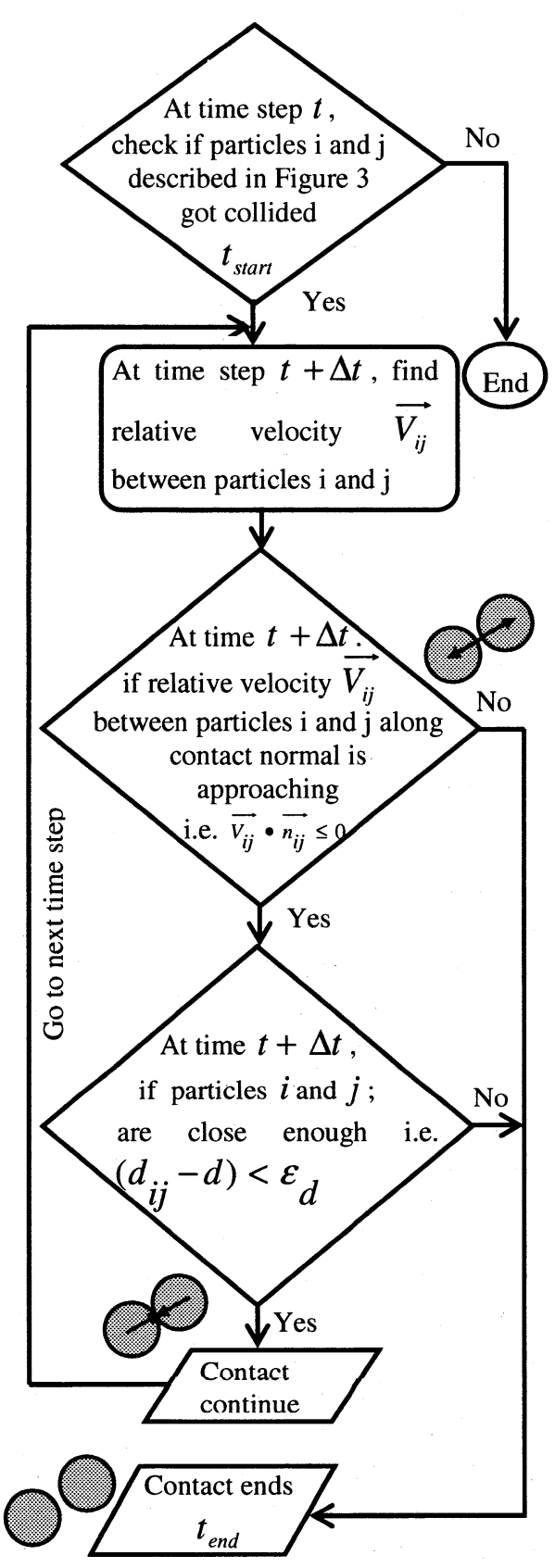

(b)

Figure 8 Flow chart for contact detection: a) Detecting collisions, and b) Finding continueing contacts and lost contacts.

in between successive time step difference ' $\Delta t$ '. It is to note that $\Delta t$ comes from the frame rate of high speed video camera, and therefore it is fixed for each shear rate and solid fraction cases. If we could use the higher frame rate of the camera (i.e. smaller $\Delta t$ ), the collision detection method could more precisely detect the collision and find the contact durations. This is because in the higher shear rates and low concentrations, the speed of particles will be higher and they interact very briefly and separate with each other instantaneously, which could not be detected if frame rate is low. It is probably because of the higher $\Delta t$ that few of the collisions are lost even by the presently formulated methodology. This will be explained in section 5 .

The streaming velocity, $V_{x}$ and transverse velocity, $V_{y}$ are calculated respectively as: 
$V_{x}=\left(X_{t+\Delta t}-X_{t}\right) / \Delta t$ and $V_{y}=\left(Y_{t+\Delta t}-Y_{t}\right) / \Delta t$ from which the change in velocity magnitude $\Delta V$ in between two successive time steps is determined. In addition, change in the direction of velocity $\Delta \psi$ for each particle is determined using the dot product of velocity vectors of that particle in two successive time steps i.e. $\Delta \psi=\cos ^{-1}\left(\hat{V}_{t+\Delta t} \cdot \hat{V}_{t}\right)$ where $\hat{V}=\vec{V} /|V|$ is the unit vector. Those particles are considered potential candidates for collision if their velocity magnitude change $\Delta V$ is greater than a threshold velocity $\varepsilon_{v}$, or if their direction change $\Delta \psi$ is greater than a threshold angle $\varepsilon_{\psi}$ on moving from the current time step $t$ to the next time step $t+\Delta t$, compared to the values from the previous time step $t-\Delta t$ to the current time step $t$. The same methodology is applied in each time step. The values of $\varepsilon_{v}$ and $\varepsilon_{\psi}$ are set different but optimized for the sparse and dense cases (Table 4). To optimize these thresholds, several test experiments were carried out for sparse to dense concentration cases, and the post collision velocity direction change and magnitude change of each colliding pairs as seen in particle tracking software were analyzed. The values less than the lowest observed values for each concentration cases were set as thresholds so as not to loose any potential partners of collision. Such a different thresholds are required because in the dense case, the magnitude of the velocity vector change after particle collision is highly diminished due to the limited space available for particles to travel. Despite this effort, few of the colliding pairs are lost, which will be described in section 5 . This limitation can be addressed in future studies.

Table 4. Different thresholds used in the collision detection methodology.

\begin{tabular}{llll}
\hline Solid fraction & $\begin{array}{l}\varepsilon_{d}(\% \text { of particle } \\
\text { diameter })\end{array}$ & $\varepsilon_{v}$ & $\mathcal{E}_{\psi}$ \\
\hline$\leq 0.48$ & $8 \%$ & $20 \%$ & $10^{\circ}$ \\
$>0.48<0.56$ & $5 \%$ & $15 \%$ & $7^{\circ}$ \\
$\geq 0.56$ & $2 \%$ & $8 \%$ & $4^{\circ}$ \\
\hline
\end{tabular}

\subsubsection{Detection of collision partners}

After detecting potential candidates for collision, the collision partners at time step $t$ are confirmed by checking the distance $d_{i j}$ between each of the potential collision candidates at the same time step $t$. If the difference of the distance between the potential collision candidate $d_{i j}$ and the particle diameter $d$ is less than some threshold distance $\varepsilon_{d}$, they are considered collision partners. The magnitude of $\varepsilon_{d}$ is optimized and set different for sparse and dense cases based on observations from several test experiments and on considering the accuracy of position data as described in section 2.2.

\subsubsection{Finding the continuity of contacts}

To know the status of collision partners in the next time frame, the relative velocity along their contact normal directions, defined by the dot product of the relative velocity vector $\vec{V}_{i j}$ and the unit normal vector $\overrightarrow{n_{i j}}$, on moving from time step $t+\Delta t$ to $t+2 . \Delta t$ is checked. If it has positive value i.e. going away from each other (separating), the contact is assumed to be lost in time step $t+\Delta t$. However, if it is negative or equal to zero i.e. if the particles are coming closer or move together, the distance between particle centers is checked in time step $t+\Delta t$. If this is within the limit of the threshold distance $\varepsilon_{d}$ defined before, that pair is considered to be still in contact; otherwise, it is considered to have lost contact at time step $t+\Delta t$. This process continues until the pair separates. The same methodology is repeated for all pairs in the shear flow at each time step, and an array of collision status listing collision partners and collision time steps is recorded as output.

\subsection{Method to find contact durations}

The time record of the first collision and that of separation is kept for all the collision pairs using the collision-detection algorithm, described in section 4.1 and shown graphically in Figures 8(a) and 8(b). The difference between the separation time $\left(t_{\text {end }}\right)$ and the time of first collision ( $\left.t_{\text {start }}\right)$ gives the contact duration $t_{c i}$ of that particular pair ('Eq. 1'). If the pairs arc collided at time step $\iota$ but are separated at time step $t+\Delta t$, the contact duration of that particular pair is $\Delta t$. However, if the particles are found to be in continuous contact at time step $t+\Delta t$, they are followed until they separate, and the difference between the time of separation and that of collision is calculated. If, for example, the pairs are separated at time $t+n \Delta t$, the contact duration will be $n \Delta t$. The average contact duration of all the pairs in all the time frames calculated using 'Eq. (2)' gives the average contact duration $t_{c}$ of that particular flow: 


$$
\begin{aligned}
t_{c i} & =\left(t_{\text {start }}-t_{\text {end }}\right) \\
t_{c} & =\frac{1}{N} \times \sum_{i=1}^{N} t_{c i}
\end{aligned}
$$

where $N$ is the total number of pairs counted for all the time frames under consideration and $t_{c i}$ is the contact duration of each pair.

\section{RESULTS AND DISCUSSIONS}

One of the important concerns of the contact detection methodology is whether the proposed method can work effectively for any combinations of shear rate and solid fractions. This question is quite important because the kinematics of dense and sparse assembly under different deformation rate will be different. Under high shear deformation rate, the change in velocity vector after collision can be considerably higher compared to the slow deformation rate for the same assembly. Considering these differences, we used different threshold values of the velocity vector change to find the potential partners of collision (Table 4). These values were optimized based on the observations in several test experiments in sparse to dense concentration cases under different deformation rate. Due care is given on the fact that in the sparse assembly under high deformation rate, the particles might make instantaneous collision for very short duration and got separated very soon so that the precision of the high speed video camera might not be sufficient to capture that collision. For this, the threshold distance to confirm the collision is assigned higher values (Table 4). In the dense and slow case on the other hand, particles have very little space to travel and hence the distance travelled by particles in each collision will be small, which can be related also with the mean separation distance or free flight time. The mean separation distance is directly proportional to the particle concentration ${ }^{10)}$ hence, lower threshold values are used for high concentration cases.

Figures 9 and 10 are presented here to show the change of velocity direction and velocity magnitude after collision, for the solid fractions of 0.40 and 0.56 respectively. In the same figures, the contacts detected by presently formulated methodology and that detected by past method where only the change in velocity direction criteria was used $^{7)}$, are also compared. It can be seen that the previous method could not accurately detect the collisions where post collision velocity direction change is small but the velocity magnitude change is higher. This feature is observed in all the shear flow under investigation irrespective of the solid fractions, however only two fraction cases are presented here to show the representative results (Figures 9 and 10). With the addition of velocity magnitude change criteria in the present study, those lost contacts could be accurately detected. Detecting this much of lost collisions is quite important especially in the experimental granular flow where getting the particle scale information is very difficult until recently. This is considered as one of the major contributions of the proposed method in the field of experimental granular flow study. It can be seen in the same figures that few of the collisions are still lost by present methodology either because of the limitations of the frame rate of high speed video camera or because of the higher thresholds. However, such lost collisions are only few percentage of the total. This limitation can be addressed in future studies.

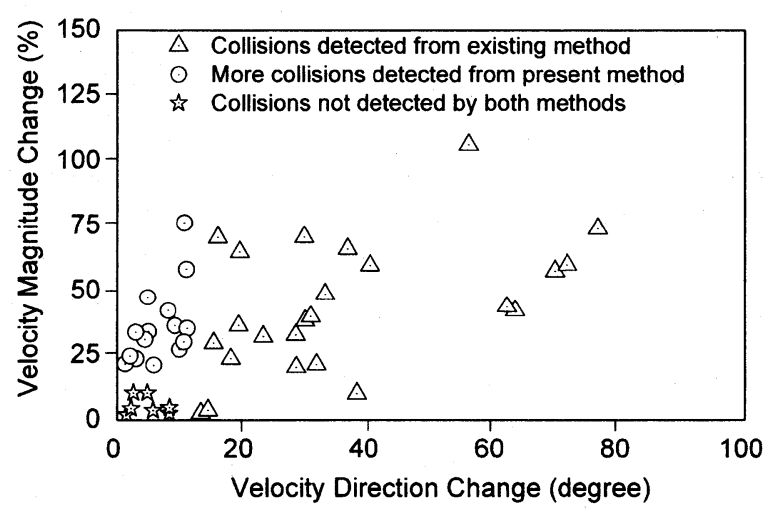

Figure 9 Change of velocity direction and velocity magnitude after collision. The proposed method could detect the contacts that are lost from the existing contact detection method (shear flow with shear rate $34.6 / \mathrm{s}$, solid fraction 0.40 ).

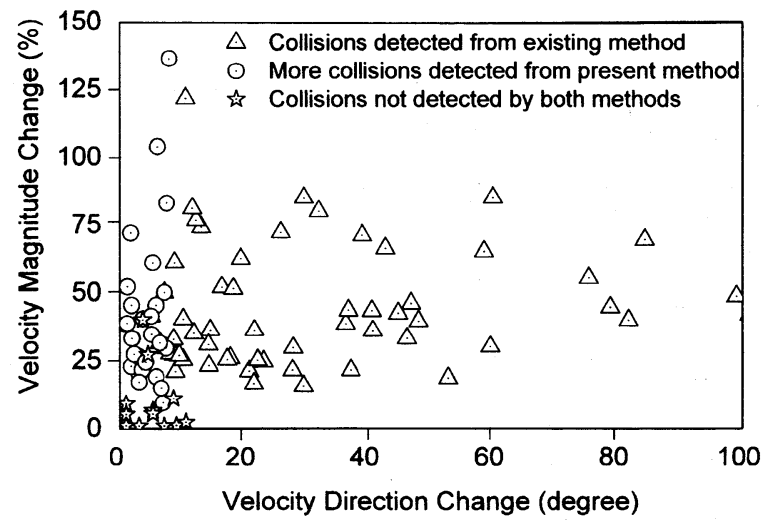

Figure 10 Change of velocity direction and velocity magnitude after collision. The proposed method could detect the contacts that are lost from the existing contact detection method (shear flow with shear rate $34.6 / \mathrm{s}$, solid fraction 0.56 ). 
To analyze the accuracy of presently proposed methodology precisely in different shear rate and solid fraction cases, the results for different combinations of shear rate and solid fractions from the algorithm are compared with the observations in the digital video data as seen in particle tracking and motion analytical software, DippMotion. To compare the results, the collisions that have been collided in the particular time step of interest are visually counted in the motion analytical software, and the counts for the same time steps from the collision detection methodology are compared. Though it is time consuming, careful visual judgment of collisions in motion analytical software ensure the usefulness of counting the collisions at least for the purpose of checking accuracy of the proposed methodology. The percentage of the collisions detected from the algorithm to the collisions observed in particle tracking software gives the accuracy of the method. The comparisons shown in Figure 11 implies that such an accuracy of the newly formulated contact detection method, ranges from almost $90 \%$ to $100 \%$. The sparse concentration cases are found relatively less accurate than the dense concentration flow, which might be partially because the former are more unstable and the fluctuations in velocity is quite high compared to the later as found by Mahmood ${ }^{11)}$. Therefore, the thresholds assigned to find the potential partners might have underestimated some of the collisions. However, Figure 11 also depicts that the accuracy level does not vary seriously with the shear rate.

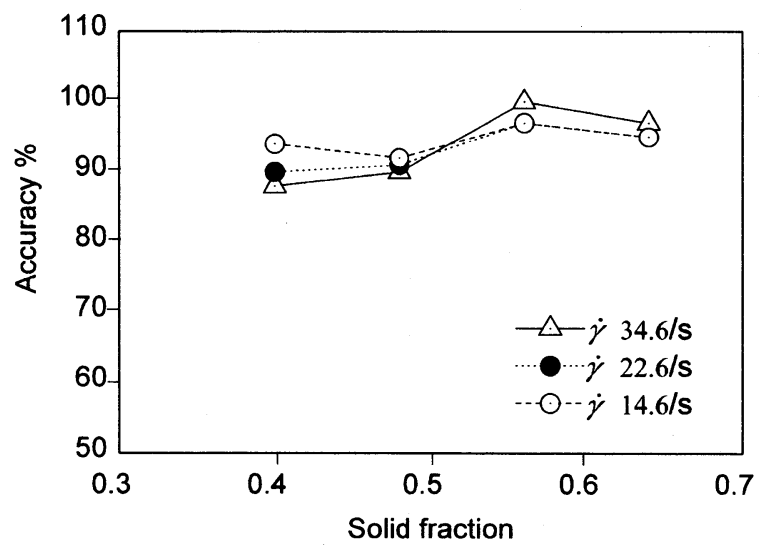

Figure 11 Accuracy of proposed method of collision detection as compared to the visual observation of digital video in particle tracking software (lines are drawn only to make it easy to follow the points).

Moreover, the methodology adopted in Blair and Kundrolli ${ }^{7}$ is applied and the results are compared with the collision detection method formulated in this study. The graph showing the detected contact percentage based on the observations on motion analytical software for both of the methods is plotted in Figure 12. The meaning of accuracy is same as it is in Figure 11. It can be seen that present methodology has quite high accuracy level compared to the previous method. Using presently formulated method the accuracy of the collision detection could be increased by almost $30 \%$ in an average. Since the collision detection methodology is extended to find the duration of contacts as well, this method can be used to find the average contact durations of inter-particle collisions in the shear flow which is particularly important to find the applicability of binary collision considerations in theoretical models based on kinetic theory of gases.

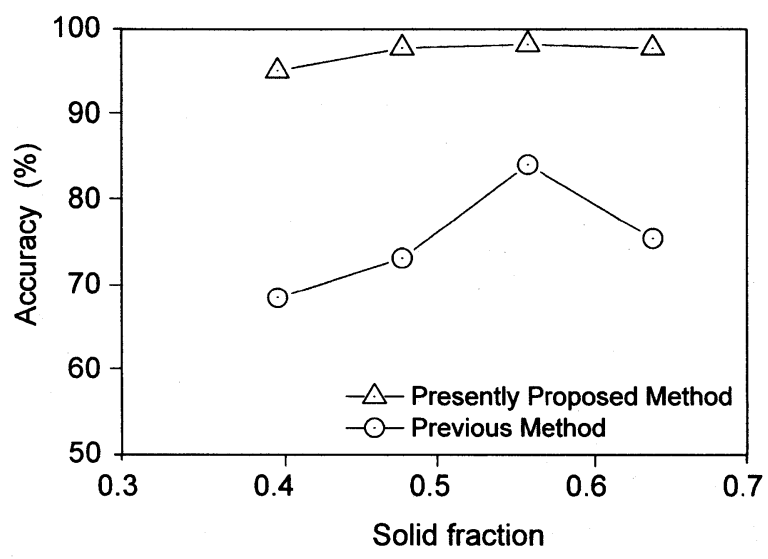

Figure 12 Comparisons of accuracy of proposed method of collision detection with the previous method ${ }^{7}$ where only the velocity direction change criteria was used (lines are drawn only to make it easy to follow the points).

One of the results of contact durations is presented here which compare the contact durations in terms of solid fraction and shear rate. It is found that when the solid fractions increases, the contact durations increases, whereas, when the shear rate is increased, it starts to decrease for the fixed solid fractions (Figure 13). Shen and Sankaran ${ }^{10)}$ also found that the average contact durations decreases with the shear rate. Present result also coincides qualitatively with the numerical simulation result of Zhang ${ }^{12)}$ where it was found that the relaxation time of contact durations is inversely proportional to the macroscopic strain rate. The consistency can also be found with the conceptual description of Campbell ${ }^{13)}$ that in denser cases, the contact duration is expected to be inversely proportional to the shear rate. These results, to some extent, validate the proposed method of finding contact durations. Therefore, the accuracy of the contact durations detected by presently 
proposed method might be reasonably good. The study can be extended to find the evolution of multiple-particle interactions in relatively high dense flows under different shear deformation rates.

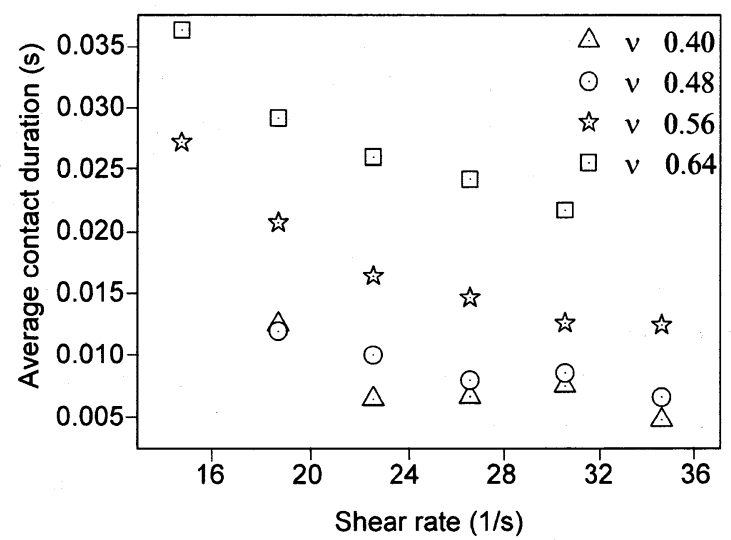

Figure 13 Variation of average contact durations of the flow with the applied shear rates for different solid fraction (V) cases.

\section{CONCLUSIONS}

Based on the observations of pre and post collision phenomena in the physical experiments, new contact detection algorithm is formulated. This method is useful also to find the collisions which continue for greater than instantaneous binary collision. Therefore it can distinguish even the binary and multi-particle collisions, which is essentially important to study the flow behavior of moderately dense to dense granular flow. Comparisons with the visual observations of the experimental data with the help of particle tracking and motion analytical software reveal that the proposed method can precisely detect the collisions with remarkable accuracy. Such a detection was possible in all the combinations of shear deformation rate and particle concentration that have been used in this study. In addition, the proposed method produces considerable increase in accuracy compared to the previous method which uses only the change in velocity direction criteria to find the potential partners of collision. Use of higher frame rate of high speed video camera and inclusion of particle rotation may increase the effectiveness of the collision detection and contact duration finding methodologies. Results of present study for the variation of contact duration with the shear rate is qualitatively consistent with the some of the past studies. Such an ability of finding the durations of each collision in the experimental shear flow is one of the unique contributions of this study.

ACKNOWLEDGEMENTS: The financial support to the first author is provided by Ministry of Education, Science, Sports and culture, Japan. This research was partially supported by the Japan Society for the Promotion of Science (JSPS), Grant-in-Aid for Scientific Research (C), Grant Number 18560482, 2006.

\section{REFERENCES}

1) Goodman, M.A., and Cowin, S.C.: A Continuum Theory for Granular Materials, Arch. Rat. Mech. And Anal., 44, 249, 1972.

2) Cowin, S.C.: A theory for the flow of Granular Materials, Powder Technology, 9, 61, 1974.

3) Cowin, S.C., and Goodman, M.A.: A Variational Principle for Granular Materials, ZAMM, 56, 281, 1976.

4) Vemuri, B. C., Chen, L., Vu-Quoc, L., Zhang, X., Walton, O.: Efficient and Accurate Collision Detection for Granular Flow Simulation, Graphical Models and Image Processing 60, pp 403-422, 1998.

5) Hockney, R. W., Eastwood, I. W.: Computer Simulation Using Particles, McGraw-Hill, New York, 1981.

6) Allen, M. P., Tildesley, D. J.: Computer Simulation of Liquids, Clarendon Press, Oxford, 1987.

7) Blair, D. L., Kundrolli, A.: Collision statistics of driven granular materials, Phys. Rev. E 67, 041301, 2003.

8) Elliot, K. E., Ahmadi, G., Kvasnak, W.: Couette flows of a granular monolayer - an experimental study, J. Non-Newtonian Fluid mech. 74, pp 89-111, 1998.

9) You, C., Zhao, H., Cai, Y., Qi, H., Xu, X.: Experimental investigation of inter-particle collision rate in particulate flow. Int. J. Multiphase Flow 30, pp 1121-1138, 2004.

10) Shen, H. H., Sankaran, B.: Internal length and time scales in a simple shear granular flow, Phys. Rev. E 70, 051308, 2004.

11) Mahmood, Z.: Measurment of particle dynamics in rapid granular shear flow, Unpublished Masters thesis, Saitama University, Japan, 47 pp, 2006.

12) Zhang, D. Z.: Evolution of enduring contacts and stress relaxation in a dense granular medium, Phys. Rev. E 71, 041303, 2005.

13) Campbell, C.: Granular shear flows at the elastic limit, J. Fluid Mech. 465, pp 261-291, 2002.

(Received April 14, 2008) 